\section{Aktionsplan Allergie ante portas}

\begin{abstract}
A llergien beeinträchtigen die Lebensqualität eines großen Teils der Bevölkerung und haben erhebliche volkswirtschaftliche Auswirkungen - diese Erkenntnis hat jetzt auch das Bundesinstitut für Risikobewertung in Berlin gewonnen und ein Aktionsprogramm gegen Allergien angekündigt. Das Institut ist dem Bundesministerium für Ernährung, Lebensmittelsicherheit und Verbraucherschutz angegliedert, seine Aufgabe ist es, gesundheitliche Risiken zu erkennen, Empfehlungen zur Risiko-
\end{abstract}

\section{Forschungsstipendium für Atemwegserkrankungen}

Die Deutsche Atemwegsliga möchte zusammen mit dem Unternehmen GlaxoSmithKline den wissenschaftlichen Nachwuchs in der Pneumologie fördern und hat ein mit 10.000 Euro ausgestattetes Forschungsstipendium für Atemwegserkrankungen ausgeschrieben. Teilnahmeberechtigt sind approbierte Ärzte bzw. junge Wissenschaftler mit einer vergleichbaren naturwissenschaftlichen Qualifikation. Die Altersgrenze liegt bei 35 Jahren. Die Stipendiaten müssen einer Klinik/ und dürfen nicht gleichzeitig durch eine andere Institution für das entsprechende Vorhaben gefördert werden. Die eingereichten Projektvorschläge sollten einen klinischen Bezug haben.

Die Bewerbungsunterlagen müssen bis zum 9. Dezember 2007 eingereicht werden und einen wissenschaftlichen Lebenslauf, eine Projektbeschreibung auf maximal fünf DIN A4 Seiten, eine Aufstellung des Klinik-/Institutsleiters, dass die für die Durchführung des Forschungsvorhabens notwendigen Voraussetzungen erfüllt sind, umfassen. Da mit dem Stipendium eine Person gefördert werden soll, dürfen die Sachkosten höchstens die Hälfte der gesamten Projektkosten ausmachen.

\section{Dr. Uta Butt}

Deutsche Atemwegsliga, Im Prinzenpalais: Burgstraße, 33175 Bad Lippspringe

Tel.: (o 2732$) 769470$

Fax: (o 2732$) 769471$

E-Mail: atemwegsliga.u.butt@t-online.de einem Institut in Deutschland angehören der Projektkosten sowie eine Bestätigung begrenzung $\mathrm{zu}$ erarbeiten und diesen Prozess zu kommunizieren. Schon im Juli hatte Bundesverbraucherschutzminister Horst Seehofer den nationalen Aktionsplan gegen Allergien erstmals angekündigt, Prof. Dr. Dr. Andreas Hensel, Präsident des Bundesinstituts für Risikobewertung, bestätigte jetzt im Rahmen einer Pressekonferenz: „Eine Bündelung aller vorhandenen Informationen zum Thema der Entstehung von Allergien und die Formulierung konkreter Handlungsoptionen ist geplant."
Als Experten aus dem klinischen Bereich sind bereits Prof. Dr. Ulrich Wahn, Berlin, und Prof. Dr. Thomas Diepgen, Heidelberg, hinzugezogen worden. Beide begrüßen die neue Initiative: Ein konzertiertes Aktionsprogramm gegen die Volkskrankheit Allergie sei überfällig. Das Bundesinstitut will in einem nächsten Schritt medizinische Fachverbände, Universitäten und andere beteiligte Kreise zu Expertengesprächen einladen, um das Thema weiter zu vertiefen. Darüber hinaus wird es Instrumente einer gezielten Risikokommunikation entwickeln, um die Verbraucher optimal zu informieren und zu schützen. es

\section{Jahrestreffen der Pollenjäger}

$Z$ u ihrer Jahrestagung 2006 trafen sich am 27. September Vorstand und Beirat der Stiftung Deutscher Polleninformationsdienst (PID) in Bad Lippspringe. Zum neuen Vorsitzenden des Beirates wurde einstimmig Dr. Thomas Kehl, Sanofi Aventis Pharma, Frankfurt/Main, gewählt. Der dreiköpfige Vorstand blieb dagegen unverändert: Dr. Horst Müsken, Bad Lippspringe, Dipl.-Met. Gunild Scheid, Deutscher Wetterdienst, Essen, sowie als Vorsitzender Prof. Dr. Karl-Chris- tian Bergmann, Allergie-Centrum Charité, Berlin.

Aktuell befasst sich der Polleninformationsdienst verstärkt mit der zunehmenden Verbreitung von Ambrosiapollen in Deutschland. Im Jahr 2008 wird die Stiftung außerdem das 8. Europäische Pollenflugsymposium ausrichten und damit zugleich ihr 25-jähriges Bestehen feiern. Weitere Informationen zur Arbeit des Polleninformationsdienstes sind unter www.pollenstiftung.de im Internet abzurufen.

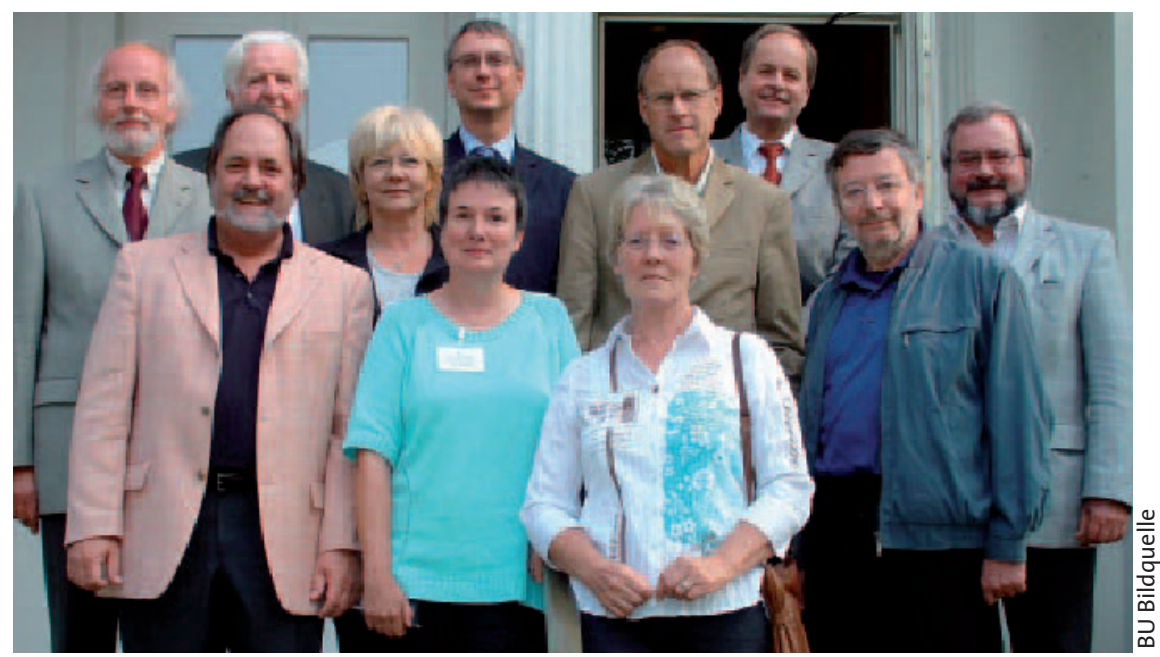

Aktueller Vorstand und Beirat der Stiftung Deutscher Polleninformationsdienst. Vordere Reihe von links nach rechts: Dr. Horst Müsken, Prof. Dr. Heidrun Behrendt, Margarete Wilhelm (PID-Geschäftsstelle), Dipl.-Met. Gunild Scheid, Dr. Thomas Höhler, Dr. Helmut Mader; hintere Reihe von links nach rechts: Dr. Klaus Bucher, Prof. Dr. Günther Forck, Dr. Thomas Kehl, Prof. Dr. Karl-Christian Bergmann, Wolfgang Jitschin 